\title{
Blowup for a Non-Newtonian Polytropic Filtration System Coupled via Nonlinear Boundary Flux
}

\author{
Zhongping Li, ${ }^{1}$ Chunlai $\mathrm{Mu}^{2}$ and Yuhuan $\mathrm{Li}^{3}$ \\ ${ }^{1}$ Department of Mathematics, China West Normal University, Nanchong 637002, China \\ ${ }^{2}$ College of Mathematics and Physics, Chongqing University, Chongqing 400044, China \\ ${ }^{3}$ Department of Mathematics, Sichuan Normal University, Chengdu 610066, China
}

Correspondence should be addressed to Chunlai Mu, chunlaimu@yahoo.com.cn

Received 18 November 2007; Accepted 1 March 2008

Recommended by Emmanuele DiBenedetto

\begin{abstract}
We study the global existence and the global nonexistence of a non-Newtonian polytropic filtration system coupled via nonlinear boundary flux. We first establish a weak comparison principle, then discuss the large time behavior of solutions by using modified upper and lower solution methods and constructing various upper and lower solutions. Necessary and sufficient conditions on the global existence of all positive (weak) solutions are obtained.
\end{abstract}

Copyright $(2008$ Zhongping Li et al. This is an open access article distributed under the Creative Commons Attribution License, which permits unrestricted use, distribution, and reproduction in any medium, provided the original work is properly cited.

\section{Introduction}

In this paper,we consider the following Neumann problem:

$$
\begin{gathered}
\left(u^{k_{1}}\right)_{t}=\Delta_{m} u, \quad\left(v^{k_{2}}\right)_{t}=\Delta_{n} v, \quad x \in \Omega, t>0, \\
\nabla_{m} u \cdot v=u^{\alpha} v^{p}, \quad \nabla_{n} v \cdot v=u^{q} v^{\beta}, \quad x \in \partial \Omega, t>0, \\
u(x, 0)=u_{0}(x), \quad v(x, 0)=v_{0}(x), \quad x \in \bar{\Omega},
\end{gathered}
$$

where $\Delta_{k} u=\operatorname{div}\left(|\nabla u|^{k-1} \nabla u\right)=\sum_{i=1}^{N}\left(|\nabla u|^{k-1} u_{x_{i}}\right)_{x_{i}}, \nabla_{k} u=\left(|\nabla u|^{k-1} u_{x_{1}}, \ldots,|\nabla u|^{k-1} u_{x_{N}}\right), \Omega \subset$ $\mathbb{R}^{N}$ is a bounded domain with smooth boundary $\partial \Omega, v$ is the outward normal vector on the boundary $\partial \Omega, k_{1}, k_{2}, m, n>0, \alpha, \beta \geq 0, p, q>0$, and $u_{0}(x), v_{0}(x) \in C^{1}(\bar{\Omega})$ are positive and satisfy the compatibility conditions.

Parabolic equations like (1.1) appear in population dynamics, chemical reactions, heat transfer, and so on. In particular, (1.1) may be used to describe the nonstationary flows in a 
porous medium of fluids with a power dependence of the tangential stress on the velocity of displacement under polytropic conditions. In this case, (1.1) are called the non-Newtonian polytropic filtration equations (see [1-6] and the references therein). For the Neuman problem (1.1)-(1.3), the local existence of solutions in time has been established, see survey in [4].

Recall the single quasilinear parabolic equation with nonlinear boundary condition

$$
\begin{aligned}
& \left(u^{k}\right)_{t}=\Delta u, \quad x \in \Omega, t>0, \\
& \frac{\partial u}{\partial v}=u^{\alpha}, \quad x \in \partial \Omega, t>0 \\
& u(x, 0)=u_{0}(x), \quad x \in \bar{\Omega},
\end{aligned}
$$

with $k>0, \alpha \geq 0$.It is known that the solutions of (1.4) exist globally if and only if $\alpha \leq k$ for $0<k \leq 1$; they exist globally if and only if $\alpha \leq(k+1) / 2$ when $k>1$ (see [7-10]).

In $[11,12], M$. Wang and S. Wang studied the following nonlinear diffusion system with nonlinear boundary conditions

$$
\begin{aligned}
& \left(u^{k_{1}}\right)_{t}=\Delta u, \quad\left(v^{k_{2}}\right)_{t}=\Delta v, \quad x \in \Omega, t>0, \\
& \frac{\partial u}{\partial v}=u^{\alpha} v^{p}, \quad \frac{\partial v}{\partial v}=u^{q} v^{\beta}, \quad x \in \partial \Omega, t>0, \\
& u(x, 0)=u_{0}(x), \quad v(x, 0)=v_{0}(x), \quad x \in \bar{\Omega},
\end{aligned}
$$

with $k_{1}, k_{2}>0, \alpha, \beta \geq 0, p, q>0$. In [11], they obtained the necessary and sufficient conditions to the global existence of solutions for $0<k_{1}, k_{2} \leq 1$. In [12], they considered the case of $k_{1}>1$ or $k_{2}>1$ and obtained the necessary and sufficient blowup conditions for the special case $\Omega=B_{R}(0)$ (the ball centered at the origin in $\mathbb{R}^{N}$ with radius $R$ ). However, for the general domain $\Omega$, they only gave some sufficient conditions to the global existence and the blowup of solutions.

In [2], Wang considered the following system with nonlinear boundary conditions:

$$
\begin{gathered}
\left(u^{k_{1}}\right)_{t}=\left(\left|u_{x}\right|^{m-1} u_{x}\right)_{x^{\prime}} \quad\left(v^{k_{2}}\right)_{t}=\left(\left|v_{x}\right|^{n-1} v_{x}\right)_{x^{\prime}} \quad x \in(0,1), t>0, \\
u_{x}(0, t)=0, \quad u_{x}(1, t)=\lambda u^{\alpha} v^{p}(1, t), \quad t>0, \\
v_{x}(0, t)=0, \quad v_{x}(1, t)=\lambda u^{q} v^{\beta}(1, t), \quad t>0, \\
u(x, 0)=u_{0}(x), \quad v(x, 0)=v_{0}(x), \quad x \in[0,1],
\end{gathered}
$$

with $\lambda>0$. They obtained the necessary and sufficient conditions on the global existence of all positive (weak) solutions.

Sun and Wang in [13] studied the nonlinear equation with nonlinear boundary condition

$$
\begin{aligned}
& \left(u^{k}\right)_{t}=\Delta_{m} u, \quad x \in \Omega, t>0, \\
& \nabla_{m} u \cdot v=u^{\alpha}, \quad x \in \partial \Omega, t>0, \\
& u(x, 0)=u_{0}(x), \quad x \in \bar{\Omega} .
\end{aligned}
$$


They proved that all positive (weak) solutions of (1.7) exist globally if and only if $\alpha \leq k$ when $k \leq m$; they exist globally if and only if $\alpha \leq m(k+1) /(m+1)$ when $k>m$.

The main purpose of this paper is to study the influence of nonlinear power exponents on the existence and nonexistence of global solutions of (1.1)-(1.3). By using upper- and lowersolution methods, we obtain the necessary and sufficient conditions on the existence of global (weak) solutions to (1.1)-(1.3). Our main results are stated as follows.

Theorem 1.1. If $k_{1} \geq m, k_{2} \geq n$, then all positive (weak) solutions of (1.1)-(1.3) exist globally if and only if $\alpha \leq m\left(k_{1}+1\right) /(m+1), \beta \leq n\left(k_{2}+1\right) /(n+1)$ and $p q \leq\left(m\left(k_{1}+1\right) /(m+1)-\alpha\right)\left(n\left(k_{2}+\right.\right.$ $1) /(n+1)-\beta)$.

Theorem 1.2. If $k_{1}<m, k_{2} \geq n$, then all positive (weak) solutions of (1.1)-(1.3) exist globally if and only if $\alpha \leq k_{1}, \beta \leq n\left(k_{2}+1\right) /(n+1)$ and $p q \leq\left(k_{1}-\alpha\right)\left(n\left(k_{2}+1\right) /(n+1)-\beta\right)$.

Theorem 1.3. If $k_{1} \geq m, k_{2}<n$, then all positive (weak) solutions of (1.1)-(1.3) exist globally if and only if $\alpha \leq m\left(k_{1}+1\right) /(m+1), \beta \leq k_{2}$ and $p q \leq\left(m\left(k_{1}+1\right) /(m+1)-\alpha\right)\left(k_{2}-\beta\right)$.

Theorem 1.4. If $k_{1}<m, k_{2}<n$, then all positive (weak) solutions of (1.1)-(1.3) exist globally if and only if $\alpha \leq k_{1}, \beta \leq k_{2}$ and $p q \leq\left(k_{1}-\alpha\right)\left(k_{2}-\beta\right)$.

Remark 1.5. If $m=n=1,0<k_{1}, k_{2} \leq 1$, the results in [11] are included in Theorem 1.4, and if $m=n=1, k_{1}>1$ or $k_{2}>1$, Theorems 1.1-1.3 improve the results of [12].

Remark 1.6. If we extend the solution to (1.6) to the interval $[-1,1]$ by symmetry, we get a solution to the same problem (1.6) with the condition at $x=0$, substituted by a condition at $x=$ $-1,-u_{x}(-1, t)=\lambda u^{\alpha} v^{p}(-1, t),-v_{x}(-1, t)=\lambda u^{q} v^{\beta}(-1, t), t>0$. Conversely, symmetric solutions to this latter problem are solutions to the original problem (1.6). The problem (1.1)-(1.3) is the more general $N$-dimensional version of the problem (1.6). Theorems 1.1-1.4 extend the results of the problem (1.6) into multidimensional case and it seems to be a natural extension of Wang [2].

Remark 1.7. If $k_{1}=k_{2}, m=n, \alpha=\beta, p=q=0$, the conclusions of Theorems 1.1 and 1.4 are consistent with those of the single equation (1.7). This paper generalizes the results of the single equation (1.7) to the system (1.1)-(1.3).

The rest of this paper is organized as follows. Some preliminaries will be given in Section 2. Theorems 1.1-1.4 will be proved in Sections 3-5, respectively.

\section{Preliminaries}

As it is well known that degenerate and singular equations need not possess classical solutions, we give a precise definition of a weak solution to (1.1)-(1.3).

Definition 2.1. Let $T>0$ and $Q_{T}=\Omega \times(0, T]$. A vector function $(u(x, t), v(x, t))$ is called a weak upper (or lower) solution to (1.1)-(1.3) in $Q_{T}$ if

(i) $u(x, t), v(x, t) \in L^{\infty}\left(0, T ; W^{1, \infty}(\Omega)\right) \cap W^{1,2}\left(0, T ; L^{2}(\Omega)\right) \cap C\left(\overline{Q_{T}}\right)$;

(ii) $(u(x, 0), v(x, 0)) \geq(\leq)\left(u_{0}(x), v_{0}(x)\right)$; 
(iii) for any positive two functions $\psi_{1}, \psi_{2} \in L^{1}\left(0, T ; W^{1,2}(\Omega)\right) \cap L^{2}\left(Q_{T}\right)$, one has

$$
\begin{aligned}
& \iint_{Q_{T}}\left[\left(u^{k_{1}}\right)_{t} \psi_{1}+\nabla_{m} u \cdot \nabla \psi_{1}\right] d x d t \geq(\leq) \int_{0}^{T} \int_{\partial \Omega} u^{\alpha} v^{p} \psi_{1} d s d t \\
& \iint_{Q_{T}}\left[\left(v^{k_{2}}\right)_{t} \psi_{2}+\nabla_{n} v \cdot \nabla \psi_{2}\right] d x d t \geq(\leq) \int_{0}^{T} \int_{\partial \Omega} u^{q} v^{\beta} \psi_{2} d s d t .
\end{aligned}
$$

In particular, $(u(x, t), v(x, t))$ is called a weak solution of (1.1)-(1.3) if it is both a weak upper and a lower solution. For every $T<\infty$, if $(u(x, t), v(x, t))$ is a solution of (1.1)-(1.3) in $Q_{T}$, we say that $(u(x, t), v(x, t))$ is global.

Next we give some preliminary propositions and lemmas.

Proposition 2.2 (comparison principle). Assume that $u_{0}, v_{0}$ are positive $C^{1}(\bar{\Omega})$ functions and $(u, v)$ is any weak solution of (1.1)-(1.3). Also assume that $(\underline{u}, \underline{v}) \geq\left(\delta_{0}, \delta_{0}\right)>0$ and $(\bar{u}, \bar{v})$ are a lower and an upper solution of (1.1)-(1.3) in $Q_{T}$, respectively, with nonlinear boundary flux $\left(\hat{\lambda u^{\alpha}} \underline{v}^{p}, \underline{\lambda} \underline{u}^{q} \underline{v}^{\beta}\right)$ and $\left(\bar{\lambda} \bar{u}^{\alpha} \bar{v}^{p}, \bar{\lambda} \bar{u}^{q} \bar{v}^{\beta}\right)$, where $0<\underline{\lambda}<1<\bar{\lambda}$. Then we have $(\bar{u}, \bar{v}) \geq(u, v) \geq(\underline{u}, \underline{v})$ in $Q_{T}$.

Proof. For small $\sigma>0$, letting $\psi_{\sigma}(z)=\min \{1, \max \{z / \sigma, 0\}\}, z \in \mathbb{R}$, and setting $\psi_{1}=\psi_{\sigma}(\underline{u}-u)$, according to the definition of upper and lower solutions, we have

$$
\begin{gathered}
\iint_{Q_{\tau}}\left[\left(\underline{u}^{k_{1}}-u^{k_{1}}\right)_{t} \psi_{\sigma}(\underline{u}-u)+\left(\nabla_{m} \underline{u}-\nabla_{m} u\right) \cdot \nabla \psi_{\sigma}(\underline{u}-u)\right] d x d t \\
\leq \int_{0}^{\tau} \int_{\partial \Omega}\left(\underline{\lambda u}^{\alpha} \underline{v}^{p}-u^{\alpha} v^{p}\right) \psi_{\sigma}(\underline{u}-u) d s d t .
\end{gathered}
$$

Define

$$
\chi(x)= \begin{cases}1, & x \geq 0 \\ 0, & x<0\end{cases}
$$

As in [14],by letting $\sigma \rightarrow 0$ we get

$$
\iint_{Q_{\tau}}\left(\underline{u}^{k_{1}}-u^{k_{1}}\right)_{t} \chi(\underline{u}-u) d x d t \leq \int_{0}^{\tau} \int_{\partial \Omega}\left(\underline{\lambda} \underline{u}^{\alpha} \underline{v}^{p}-u^{\alpha} v^{p}\right) \chi(\underline{u}-u) d s d t,
$$

that is,

$$
\left.\int_{\Omega}\left(\underline{u}^{k_{1}}-u^{k_{1}}\right)_{+}\right|_{t=\tau} d x \leq \int_{0}^{\tau} \int_{\partial \Omega}\left(\underline{\lambda u}^{\alpha} \underline{v}^{p}-u^{\alpha} v^{p}\right)_{+} d s d t
$$

where $W_{+}=\max \{W, 0\}$. Similarly, we have

$$
\left.\int_{\Omega}\left(\underline{v}^{k_{2}}-v^{k_{2}}\right)_{+}\right|_{t=\tau} d x \leq \int_{0}^{\tau} \int_{\partial \Omega}\left(\underline{\lambda u^{q}} \underline{v}^{\beta}-u^{q} v^{\beta}\right)_{+} d s d t .
$$


Since $\underline{\underline{\lambda}}<1, \underline{u}, \underline{v} \geq \delta_{0}>0$ and $\underline{u}(x, 0) \leq u_{0}(x), \underline{v}(x, 0) \leq u_{0}(x)$, it follows from the continuity of $\underline{u}, \underline{v}, u$ and $\bar{v}$ that there exists a $\tau>0$ sufficiently small such that $\underline{\lambda u^{\alpha}} \underline{v}^{p} \leq u^{\alpha} v^{p}, \underline{\lambda u^{q}} \underline{v}^{\beta} \leq u^{q} v^{\beta}$ for $(x, t) \in Q_{\tau}$. It follows from $(2.5)$ and $(2.6)$ that $(u, v) \geq(\underline{u}, \underline{v})$ in $Q_{\tau}$.

Denote $\tau^{*}=\sup \left\{\tau \in[0, T]: \underline{u}(x, t) \leq u(x, t), \underline{v}(x, t) \leq v(x, t)\right.$ for all $\left.(x, t) \in \overline{Q_{\tau}}\right\}$. We claim that $\tau^{*}=T$. Otherwise, from the continuity of $\underline{u}, \underline{v}, u$ and $v$ there exists $\epsilon>0$ such that $\tau^{*}+\epsilon<T$ and $\underline{\lambda u}^{\alpha} \underline{v}^{p} \leq u^{\alpha} v^{p}, \underline{\lambda u}^{q} \underline{v}^{\beta} \leq u^{q} v^{\beta}$ for all $(x, t) \in \overline{Q_{\tau^{*}+\epsilon}}$. By (2.5) and (2.6) we obtain

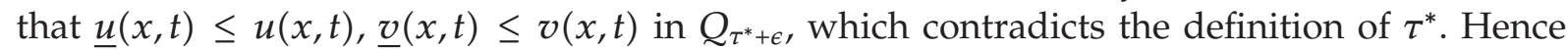
$(\underline{u}, \underline{v}) \leq(u, v)$ for all $(x, t) \in Q_{T}$.

Obviously, $\delta=\min \left\{\min _{\bar{\Omega}} u_{0}(x), \min _{\bar{\Omega}} v_{0}(x)\right\}>0$ is a lower solution of (1.1)-(1.3) in $Q_{T}$. Therefore, $u, v \geq \delta>0$ in $Q_{T}$. Using this fact, as in the above proof we can prove that $(\bar{u}, \bar{v}) \geq(u, v)$ in $Q_{T}$.

For convenience, we denote $0<\underline{\imath}<1<\bar{\lambda}$, which are fixed constants, and let $\delta=$ $\min \left\{\min _{\bar{\Omega}} u_{0}(x), \min _{\bar{\Omega}} v_{0}(x)\right\}>0$.

Proposition 2.3. Assume $k_{1} \geq m, k_{2} \geq n$ and that $\alpha>m\left(k_{1}+1\right) /(m+1)$ or $\beta>n\left(k_{2}+1\right) /(n+1)$ holds. Then the solutions of (1.1)-(1.3) blow up in finite time.

Proof. Without loss of generality, assume $\alpha>m\left(k_{1}+1\right) /(m+1)$. Consider the single equation

$$
\begin{aligned}
\left(w^{k_{1}}\right)_{t}=\nabla_{m} w, & x \in \Omega, t>0, \\
\nabla_{m} w \cdot \mathcal{v} & =\delta^{p} w^{\alpha}, \quad x \in \partial \Omega, t>0, \\
w(x, 0) & =u_{0}(x), \quad x \in \bar{\Omega} .
\end{aligned}
$$

We know from [13] that $w$ blows up in finite time. Since $v \geq \delta$, by the comparison principle, $(w, \delta)$ is a lower solution of (1.1)-(1.3) and $(u, v)$ blows up in finite time if $\alpha>m\left(k_{1}+1\right) /(m+$ $1)$.

The following propositions can be proved in the similar procedure.

Proposition 2.4. Assume $k_{1}<m, k_{2} \geq n$ and that $\alpha>k_{1}$ or $\beta>n\left(k_{2}+1\right) /(n+1)$ holds. Then the solutions of (1.1)-(1.3) blow up in finite time.

Proposition 2.5. Assume $k_{1} \geq m, k_{2}<n$ and that $\alpha>m\left(k_{1}+1\right) /(m+1)$ or $\beta>k_{2}$ holds. Then the solutions of (1.1)-(1.3) blow up in finite time.

Proposition 2.6. Assume $k_{1}<m, k_{2}<n$ and that $\alpha>k_{1}$ or $\beta>k_{2}$ holds. Then the solutions of (1.1)-(1.3) blow up in finite time.

Let $\varphi_{k}(x)(k=m, n)$ be the first eigenfunction of

$$
-\Delta_{k} \varphi=\lambda \varphi^{k}(x) \text { in } \Omega, \quad \varphi_{k}(x)=0 \text { on } \partial \Omega
$$

with the first eigenvalue $\lambda_{k}$, normalized by $\left\|\varphi_{k}(x)\right\|_{\infty}=1$, then $\lambda_{k}>0, \varphi_{k}(x)>0$ in $\Omega$ and $\varphi_{k}(x) \in W_{0}^{1, k+1} \cap C^{1}(\Omega)$ and $\partial \varphi_{k}(x) / \partial v<0$ on $\partial \Omega$ (see [15-17]). 
Thus there exist some positive constants $A_{k}, B_{k}, C_{k}, D_{k}$ such that

$$
A_{k} \leq-\frac{\partial \varphi_{k}(x)}{\partial \mathcal{v}} \leq B_{k}, \quad\left|\nabla \varphi_{k}(x)\right| \geq C_{k}, \quad x \in \partial \Omega ; \quad\left|\nabla \varphi_{k}(x)\right| \leq D_{k}, \quad x \in \bar{\Omega}
$$

We have also $\left|\nabla \varphi_{k}(x)\right| \geq E_{k}$ provided $x \in\left\{x \in \Omega: \operatorname{dist}(x, \partial \Omega) \leq \varepsilon_{k}\right\}$ with $E_{k}=C_{k} / 2$ and some positive constant $\varepsilon_{k}$. For the fixed $\varepsilon_{k}$, there exists a positive constant $F_{k}$ such that $\varphi_{k}(x) \geq F_{k}$ if $x \in\left\{x \in \Omega: \operatorname{dist}(x, \partial \Omega)>\varepsilon_{k}\right\}$.

At the end of this section, we describe two simple lemmas without proofs.

Lemma 2.7. Suppose that positive constants $A, B, C, D$ satisfy $A B<C D$, then for any two positive constants $a, b$, there exist two positive constants $l_{1}, l_{2}$ such that $a l_{1}^{C}>l_{2}^{A}$ and $b l_{2}^{D}>l_{1}^{B}$.

Lemma 2.8. For any constant $j \geq 0$, there exist positive constants $f_{i}(j)(i=1,2)$ which depend only on $j$ and $\varphi(x)$, such that

$$
f_{1}(j)\left(\varphi(x)+s^{j}\right) \leq(\varphi(x)+s)^{j} \leq f_{2}(j)\left(\varphi(x)+s^{j}\right) \quad \forall s \geq 1
$$

where $\varphi(x)$ is a positive bounded function.

\section{Proof of Theorem 1.1}

Lemma 3.1. Suppose $k_{1} \geq m, k_{2} \geq n, \alpha \leq m\left(k_{1}+1\right) /(m+1), \beta \leq n\left(k_{2}+1\right) /(n+1)$, $p q \leq$ $\left(m\left(k_{1}+1\right) /(m+1)-\alpha\right)\left(n\left(k_{2}+1\right) /(n+1)-\beta\right)$. Then all positive solutions of (1.1)-(1.3) exist globally.

Proof. Construct

$$
\begin{aligned}
& \bar{u}(x, t)=e^{l_{1} t}\left(M+\bar{\lambda}^{-1 / m} e^{-L_{1} \varphi_{m} e^{\left(k_{1}-m\right) l_{1} t /(m+1)}}(2 M)^{(p+\alpha) / m} L_{1}^{-1}\left(A_{m} c_{m}^{m-1}\right)^{-1 / m}\right), \\
& \bar{v}(x, t)=e^{l_{2} t}\left(M+\bar{\lambda}^{-1 / n} e^{-L_{2} \varphi_{n} e^{\left(k_{2}-n\right) l_{2} t /(n+1)}}(2 M)^{(q+\beta) / n} L_{2}^{-1}\left(A_{n} c_{n}^{n-1}\right)^{-1 / n}\right),
\end{aligned}
$$

where $c_{m}=C_{m}$ if $m \geq 1, c_{m}=D_{m}$ if $m<1$, and $c_{n}=C_{n}$ if $n \geq 1, c_{n}=D_{n}$ if $n<1, \varphi_{m}$, $\varphi_{n}, A_{m}, A_{n}, C_{m}, C_{n}, D_{m}, D_{n}$ are defined in (2.8) and (2.9), $l_{1}, l_{2}$ are positive constants to be determined, $M=\max \left\{1,\left\|u_{0}\right\|_{\infty},\left\|v_{0}\right\|_{\infty}\right\}$ and

$$
\begin{aligned}
& L_{1}=\bar{\lambda}^{-1 / m} \max \left\{\frac{k_{1}-m}{m+1} 2^{(p+\alpha+m) / m} M^{(p+\alpha-m) / m}\left(A_{m} c_{m}^{m-1}\right)^{-1 / m}, 2^{(p+\alpha) / m} M^{(p+\alpha-m) / m}\left(A_{m} c_{m}^{m-1}\right)^{-1 / m}\right\}, \\
& L_{2}=\bar{\lambda}^{-1 / n} \max \left\{\frac{k_{2}-n}{n+1} 2^{(q+\beta+n) / n} M^{(q+\beta-n) / n}\left(A_{n} c_{n}^{n-1}\right)^{-1 / n}, 2^{(q+\beta) / n} M^{(q+\beta-n) / n}\left(A_{n} c_{n}^{n-1}\right)^{-1 / n}\right\} .
\end{aligned}
$$


We know that $-L_{1} \varphi_{m} e^{\left(k_{1}-m\right) l_{1} t /(m+1)} e^{-L_{1} \varphi_{m} e^{\left(k_{1}-m\right) l_{1} t /(m+1)}} \geq-e^{-1}$ since $-y e^{-y} \geq-e^{-1}$ for any $y>0$. Thus for $(x, t) \in \Omega \times \mathbb{R}^{+}$, a simple computation shows

$$
\begin{aligned}
\left(\bar{u}^{k_{1}}\right)_{t}= & k_{1} l_{1} e^{k_{1} l_{1} t}\left(M+\bar{\lambda}^{-1 / m} e^{-L_{1} \varphi_{m} e^{\left(k_{1}-m\right) l_{1} t /(m+1)}}(2 M)^{(p+\alpha) / m} L_{1}^{-1}\left(A_{m} c_{m}^{m-1}\right)^{-1 / m}\right)^{k_{1}} \\
& +k_{1} e^{k_{1} l_{1} t}\left(M+\bar{\lambda}^{-1 / m} e^{-L_{1} \varphi_{m} e^{\left(k_{1}-m\right) l_{1} t /(m+1)}}(2 M)^{(p+\alpha) / m} L_{1}^{-1}\left(A_{m} c_{m}^{m-1}\right)^{-1 / m}\right)^{k_{1}-1} \\
& \times \bar{\lambda}^{-1 / m}(2 M)^{(p+\alpha) / m} L_{1}^{-1}\left(A_{m} c_{m}^{m-1}\right)^{-1 / m} \frac{\left(k_{1}-m\right) l_{1}}{m+1}\left(-L_{1} \varphi_{m}\right) e^{\left(k_{1}-m\right) l_{1} t /(m+1)} e^{-L_{1} \varphi_{m} e^{\left(k_{1}-m\right) l_{1} t /(m+1)}} \\
\geq & \frac{1}{2} k_{1} l_{1} e^{k_{1} l_{1} t} .
\end{aligned}
$$

In addition, we have

$$
\begin{aligned}
\Delta_{m} \bar{u} \leq & \overline{\mathcal{\lambda}} \lambda_{m}(2 M)^{p+\alpha}\left(A_{m} c_{m}^{m-1}\right)^{-1} \varphi_{m}^{m} e^{m l_{1} t} e^{m\left(k_{1}-m\right) l_{1} t / m} e^{-L_{1} m \varphi_{m} e^{\left(k_{1}-m\right) l_{1} t /(m+1)}} \\
& +\bar{\lambda} L_{1} m(2 M)^{p+\alpha}\left(A_{m} c_{m}^{m-1}\right)^{-1} e^{k_{1} l_{1} t} e^{-L_{1} m \varphi_{m} e^{\left(k_{1}-m\right) l_{1} t /(m+1)}}\left|\nabla \varphi_{m}\right|^{m+1} \\
\leq & \bar{\lambda}\left(\lambda_{m}+L_{1} m D_{m}^{m+1}\right)(2 M)^{p+\alpha}\left(A_{m} c_{m}^{m-1}\right)^{-1} e^{k_{1} l_{1} t} .
\end{aligned}
$$

Similarly, we can get

$$
\left(\bar{v}^{k_{2}}\right)_{t} \geq \frac{1}{2} k_{2} l_{2} e^{k_{2} l_{2} t}, \quad \Delta_{n} \bar{v} \leq \bar{\lambda}\left(\lambda_{n}+L_{2} n D_{n}^{n+1}\right)(2 M)^{q+\beta}\left(A_{n} c_{n}^{n-1}\right)^{-1} e^{k_{2} l_{2} t}
$$

Noting $\varphi_{m}=\varphi_{n}=0$ on $\partial \Omega$, we have on the boundary that

$$
\begin{aligned}
& \nabla_{m} \bar{u} \cdot \mathcal{v} \geq \bar{\lambda}(2 M)^{p+\alpha} e^{m\left(k_{1}+1\right) l_{1} t /(m+1)}, \quad \nabla_{n} \bar{v} \cdot v \geq \overline{\mathcal{\lambda}}(2 M)^{q+\beta} e^{n\left(k_{2}+1\right) l_{2} t /(n+1)} ; \\
& \bar{u}^{\alpha} \bar{v}^{p} \leq(2 M)^{p+\alpha} e^{\left(\alpha l_{1}+p l_{2}\right) t}, \quad \bar{u}^{q} \bar{v}^{\beta} \leq(2 M)^{q+\beta} e^{\left(q l_{1}+\beta l_{2}\right) t} .
\end{aligned}
$$

Since $p q \leq\left(m\left(k_{1}+1\right) /(m+1)-\alpha\right)\left(n\left(k_{2}+1\right) /(n+1)-\beta\right)$, there exist constants $l_{1}, l_{2}$ large such that

$$
\begin{gathered}
\frac{m\left(k_{1}+1\right) l_{1}}{m+1} \geq \alpha l_{1}+p l_{2}, \quad \frac{n\left(k_{2}+1\right) l_{2}}{n+1} \geq q l_{1}+\beta l_{2} \\
l_{1} \geq 2 \bar{\lambda}\left(\lambda_{m}+L_{1} m D_{m}^{m+1}\right)(2 M)^{p+\alpha}\left(k_{1} A_{m} c_{m}^{m-1}\right)^{-1} \\
l_{2} \geq 2 \bar{\lambda}\left(\lambda_{n}+L_{2} n D_{n}^{n+1}\right)(2 M)^{q+\beta}\left(k_{2} A_{n} c_{n}^{n-1}\right)^{-1} .
\end{gathered}
$$

By (3.3)-(3.7), we know that $(\bar{u}, \bar{v})$ is a global upper solution of (1.1)-(1.3). The global existence of solutions to (1.1)-(1.3) follows from the comparison principle.

Lemma 3.2. Suppose $k_{1} \geq m, k_{2} \geq n, \alpha \leq m\left(k_{1}+1\right) /(m+1), \beta \leq n\left(k_{2}+1\right) /(n+1)$, pq > $\left(m\left(k_{1}+1\right) /(m+1)-\alpha\right)\left(n\left(k_{2}+1\right) /(n+1)-\beta\right)$. Then all positive solutions of (1.1)-(1.3) blow up in finite time. 
Proof.

Case 1. $k_{1}>m, k_{2}>n$. Let $d_{m}=C_{m}$ if $m<1, d_{m}=D_{m}$ if $m \geq 1$, and $d_{n}=C_{n}$ if $n<1, d_{n}=D_{n}$ if $n \geq 1$. In light of $p q>\left(m\left(k_{1}+1\right) /(m+1)-\alpha\right)\left(n\left(k_{2}+1\right) /(n+1)-\beta\right)$, we choose $l_{1}, l_{2}$ such that

$$
\frac{m}{m+1} \leq p l_{2}-\left(\frac{m\left(k_{1}+1\right)}{m+1}-\alpha\right) l_{1}, \quad \frac{n}{n+1} \leq q l_{1}-\left(\frac{n\left(k_{2}+1\right)}{n+1}-\beta\right) l_{2} .
$$

For the above $l_{1}, l_{2}$, we set $\underline{u}=\left(1 /(b-c t)^{l_{1}}\right) e^{-a \varphi_{m}(x) /(b-c t)^{r_{1}}}, \underline{v}=\left(1 /(b-c t)^{l_{2}}\right) e^{-a \varphi_{n}(x) /(b-c t)^{r_{2}}}$, where $r_{1}=\left(\left(k_{1}-m\right) l_{1}+1\right) /(m+1), r_{2}=\left(\left(k_{2}-n\right) l_{2}+1\right) /(n+1), b=\max \left\{1, \delta^{-1 / l_{1}}, \delta^{-1 / l_{2}}\right\}$, and

$$
\begin{aligned}
& a=\min \left\{1, \underline{\Lambda}^{1 / m}\left(B_{m} d_{m}^{m-1}\right)^{-1 / m} b^{-\left(\alpha l_{1}+p l_{2}\right) / m}, \underline{\Lambda}^{1 / n}\left(B_{n} d_{n}^{n-1}\right)^{-1 / n} b^{-\left(q l_{1}+\beta l_{2}\right) / n}\right\}, \\
& c=\min \left\{\frac{m a^{m+1} E_{m}^{m+1}}{k_{1} l_{1}}, \frac{n a^{n+1} E_{n}^{n+1}}{k_{2} l_{2}}, \frac{\lambda_{m}\left(k_{1}-m\right) a^{m+1} F_{m}^{m+1}}{k_{1} l_{1}}, \frac{\lambda_{n}\left(k_{2}-n\right) a^{n+1} F_{n}^{n+1}}{k_{2} l_{2}}\right\} .
\end{aligned}
$$

By a direct computation, for $x \in \Omega, 0<t<c / b$, we obtain that

$$
\begin{gathered}
\left(\underline{u}^{k_{1}}\right)_{t} \leq k_{1} l_{1} c e^{-a k_{1} \varphi_{m}(x) /(b-c t)^{r_{1}}}(b-c t)^{-\left(k_{1} l_{1}+1\right)}, \\
\Delta_{m} \underline{u}=\frac{\lambda_{m} a^{m} \varphi_{m}^{m} e^{-a m \varphi_{m}(x) /(b-c t)^{r_{1}}}}{(b-c t)^{m\left(l_{1}+r_{1}\right)}}+\frac{m a^{m+1} e^{-a m \varphi_{m}(x) /(b-c t)^{r_{1}}}\left|\nabla \varphi_{m}\right|^{m+1}}{(b-c t)^{m\left(l_{1}+r_{1}\right)+r_{1}}} .
\end{gathered}
$$

If $x \in \Omega_{1}=\left\{x \in \Omega: \operatorname{dist}(x, \partial \Omega)>\varepsilon_{m}\right\}$, we have $\varphi_{m} \geq F_{m}$, and thus

$$
\Delta_{m} \underline{u} \geq \frac{\lambda_{m} a^{m} F_{m}^{m} e^{-a m \varphi_{m}(x) /(b-c t)^{r_{1}}}}{(b-c t)^{m\left(l_{1}+r_{1}\right)}} .
$$

On the other hand, since $-y e^{-y} \geq-e^{-1}$ for any $y>0$, we have

$$
\left(\underline{u}^{k_{1}}\right)_{t} \leq k_{1} l_{1} c e^{-a k_{1} \varphi_{m}(x) /(b-c t)^{r_{1}}}(b-c t)^{-\left(k_{1} l_{1}+1\right)}=\frac{k_{1} l_{1} c e^{-a m \varphi_{m}(x) /(b-c t)^{r_{1}}}}{a\left(k_{1}-m\right) F_{m} e(b-c t)^{m\left(l_{1}+r_{1}\right)}} .
$$

We have by (3.10), (3.13), and (3.14) that $\left(\underline{u}^{k_{1}}\right)_{t} \leq \Delta_{m} \underline{u}$ for $(x, t) \in \Omega_{1} \times(0, b / c)$.

If $x \in \Omega_{2}=\left\{x \in \Omega: \operatorname{dist}(x, \partial \Omega) \leq \varepsilon_{m}\right\}$, then $\left|\nabla \varphi_{m}\right| \geq E_{m}$, and hence

$$
\Delta_{m} \underline{u} \geq \frac{m a^{m+1} E_{m}^{m+1} e^{-a k_{1} \varphi_{m}(x) /(b-c t)^{r_{1}}}}{(b-c t)^{m\left(l_{1}+r_{1}\right)+r_{1}}}=\frac{m a^{m+1} E_{m}^{m+1} e^{-a k_{1} \varphi_{m}(x) /(b-c t)^{r_{1}}}}{(b-c t)^{k_{1} l_{1}+1}} .
$$

We follow from (3.10), (3.11), and (3.15) that $\left(\underline{u}^{k_{1}}\right)_{t} \leq \Delta_{m} \underline{u}$ for $(x, t) \in \Omega_{2} \times(0, b / c)$. 
Similarly, we can get $\left(\underline{v}^{k_{2}}\right)_{t} \leq \Delta_{n} \underline{v}$ for $(x, t) \in \Omega \times(0, b / c)$ also.

We have on the boundary that

$$
\begin{gathered}
\nabla_{m} \underline{u} \cdot \mathcal{v}=\frac{a^{m}\left|\nabla \varphi_{m}\right|^{m-1} e^{-a m \varphi_{m}(x) /(b-c t)^{r_{1}}}\left(-\partial \varphi_{m} / \partial \mathcal{v}\right)}{(b-c t)^{m\left(l_{1}+r_{1}\right)}} \leq \frac{a^{m} B_{m} d_{m}^{m-1}}{(b-c t)^{m\left(l_{1}+r_{1}\right)}} \\
\nabla_{n} \underline{v} \cdot \mathcal{v}=\frac{a^{n}\left|\nabla \varphi_{n}\right|^{n-1} e^{-a n \varphi_{n}(x) /(b-c t)^{r_{2}}}\left(-\partial \varphi_{n} / \partial v\right)}{(b-c t)^{n\left(l_{2}+r_{2}\right)}} \leq \frac{a^{n} B_{n} d_{n}^{n-1}}{(b-c t)^{n\left(l_{2}+r_{2}\right)}} \\
\underline{u}^{\alpha} \underline{v}^{p}=\frac{1}{(b-c t)^{\alpha l_{1}+p l_{2}}}, \quad \underline{u}^{q} \underline{v}^{\beta}=\frac{1}{(b-c t)^{q l_{1}+\beta l_{2}}} .
\end{gathered}
$$

Moreover, by (3.8) we have that

$$
m\left(l_{1}+r_{1}\right) \leq \alpha l_{1}+p l_{2}, \quad n\left(l_{2}+r_{2}\right) \leq q l_{1}+\beta l_{2}
$$

Equations (3.9), (3.16)-(3.18) imply that $\nabla_{m} \underline{u} \cdot \mathcal{v} \leq \underline{\lambda}^{\alpha} \underline{v}^{p}, \nabla_{n} \underline{v} \cdot \mathcal{v} \leq \underline{\lambda}^{q} \underline{v}^{\beta}$ on $\partial \Omega$. Therefore $(\underline{u}, \underline{v})$ is a lower solution of $(1.1)-(1.3)$.

Case 2. $k_{1}>m, k_{2}=n$. Set $\underline{u}$ as above with $\underline{v}=\left(1 /(b-c t)^{l_{2}}\right) e^{-a \varphi_{n}(x) /(b-c t)^{1 / n}}$.

Case 3. $k_{1}=m, k_{2}>n$. Set $\underline{v}$ as above with $\underline{u}=\left(1 /(b-c t)^{l_{1}}\right) e^{-a \varphi_{m}(x) /(b-c t)^{1 / m}}$.

Case 4. $k_{1}=m, k_{2}=n$. Set $\underline{u}=\left(1 /(b-c t)^{l_{1}}\right) e^{-a \varphi_{m}(x) /(b-c t)^{1 / m}}, \underline{v}=\left(1 /(b-c t)^{l_{2}}\right) e^{-a \varphi_{n}(x) /(b-c t)^{1 / n}}$.

By similar arguments, we conform that $(\underline{u}, \underline{v})$ is a lower solution of (1.1)-(1.3), which blows up in finite time. We know by the comparison principle that the solution $(u, v)$ blows up in finite time.

We get the proof of Theorem 1.1 by combining Proposition 2.3 and Lemmas 3.1 and 3.2.

\section{Proof of Theorems 1.2 and 1.3}

Lemma 4.1. Suppose $k_{1}<m, k_{2} \geq n, \alpha \leq k_{1}, \beta \leq n\left(k_{2}+1\right) /(n+1)$ with $p q \leq\left(k_{1}-\alpha\right)\left(n\left(k_{2}+\right.\right.$ $1) /(n+1)-\beta)$. Then all positive solutions of $(1.1)-(1.3)$ exist globally.

Proof. Take

$$
\begin{aligned}
& \bar{u}(x, t)=R_{1} e^{l_{1} t} \log \left(\left(1-\varphi_{m}(x)\right) e^{\left(k_{1}-m\right) l_{1} t / m}+R_{2}\right), \\
& \bar{v}(x, t)=e^{l_{2} t}\left(M+\bar{\lambda}^{1 / n} e^{-L \varphi_{n}(x) e^{\left(k_{2}-n\right) l_{2} t /(n+1)}}(2 M)^{\left(k_{2}+1\right) /(n+1)} L^{-1}\left(A_{n} c_{n}^{n-1}\right)^{-1 / n}\right)
\end{aligned}
$$

for $(x, t) \in \bar{\Omega} \times \mathbb{R}^{+}$, where $c_{n}=C_{n}$ if $n \geq 1, c_{n}=D_{n}$ if $n<1, R_{2}$ satisfying $R_{2} \log R_{2} \geq 2\left(m-k_{1}\right) / m$, and constants $R_{1}, M, L, l_{1}, l_{2}$ are to be determined. By performing direct calculations, we have, 
for $(x, t) \in \Omega \times \mathbb{R}^{+}$,

$$
\begin{gathered}
\left(\bar{u}^{k_{1}}\right)_{t} \geq \frac{k_{1} l_{1}}{2} R^{k_{1}} e^{k_{1} l_{1} t}\left(\log \left(\left(1-\varphi_{m}(x)\right) e^{\left(k_{1}-m\right) l_{1} t / m}+R_{2}\right)\right)^{k_{1}} \geq \frac{k_{1} l_{1}}{2} R^{k_{1}} e^{k_{1} l_{1} t}\left(\log R_{2}\right)^{k_{1}}, \\
\Delta_{m} \bar{u}=\sum_{i=1}^{N}\left(\frac{R_{1}^{m} e^{k_{1} l_{1} t}\left(-\left|\nabla \varphi_{m}(x)\right|^{m-1} \varphi_{m_{x_{i}}}\right)}{\left(\left(1-\varphi_{m}(x)\right) e^{\left(k_{1}-m\right) l_{1} t / m}+R_{2}\right)^{m}}\right)_{x_{i}} \leq \frac{\lambda_{m} R_{1}^{m} e^{k_{1} l_{1} t}}{R_{2}^{m}} .
\end{gathered}
$$

By setting $c_{m}=C_{m}$ if $m \geq 1, c_{m}=D_{m}$ if $m<1$, we have on the boundary that

$$
\begin{gathered}
\nabla_{m} \bar{u} \cdot \mathcal{v} \geq \frac{R_{1}^{m} e^{k_{1} l_{1} t} c_{m}^{m-1} A_{m}}{\left(1+R_{2}\right)^{m}}, \quad \nabla_{n} \bar{v} \cdot \mathcal{v} \geq \overline{\mathcal{\lambda}}(2 M)^{n\left(k_{2}+1\right) /(n+1)} e^{n\left(k_{2}+1\right) l_{2} t /(n+1)} ; \\
\bar{u}^{\alpha} \bar{v}^{p} \leq\left(R_{1} \log \left(1+R_{2}\right)\right)^{\alpha}(2 M)^{p} e^{\alpha l_{1} t+p l_{2} t}, \quad \bar{u}^{q} \bar{v}^{\beta} \leq\left(R_{1} \log \left(1+R_{2}\right)\right)^{q}(2 M)^{\beta} e^{q l_{1} t+\beta l_{2} t} .
\end{gathered}
$$

Since $p q<(m-\alpha)\left(n\left(k_{2}+1\right) /(n+1)-\beta\right)$, by Lemma 2.7 there exist two positive constants $R_{1}, M$ such that $R_{1} \log R_{2} \geq \max \left\{1,\left\|u_{0}\right\|_{\infty}\right\}, M \geq\left\{1,\left\|u_{0}\right\|_{\infty}\right\}$, and

$$
\begin{aligned}
& R_{1}^{m-\alpha} \geq \bar{\lambda}(2 M)^{p}\left(\log \left(1+R_{2}\right)\right)^{\alpha}\left(1+R_{2}\right)^{m}\left(c_{m}^{m-1} A_{m}\right)^{-1}, \\
& (2 M)^{n\left(k_{2}+1\right) /(n+1)-\beta} \geq R_{1}^{q}\left(\log \left(1+R_{2}\right)\right)^{q} .
\end{aligned}
$$

Set $L=\bar{\lambda}^{-1 / n} \max \left\{\left(\left(k_{2}-n\right) /(n+1)\right) 2^{\left(k_{2}+n+2\right) /(n+1)} M^{\left(k_{2}-n\right) /(n+1)}\left(A_{n} c_{n}^{n-1}\right)^{-1 / n}, 2^{\left(k_{2}+1\right) /(n+1)} M^{\left(k_{2}-n\right) /(n+1)}\right.$ $\left.\times\left(A_{n} c_{n}^{n-1}\right)^{-1 / n}\right\}$. By arguments in Lemma 3.1,for $(x, t) \in \Omega \times \mathbb{R}^{+}$, we have

$$
\left(\bar{v}^{k_{2}}\right)_{t} \geq \frac{1}{2} k_{2} l_{2} e^{k_{2} l_{2} t}, \quad \Delta_{n} \bar{v} \leq \bar{\lambda}\left(\lambda_{n}+\operatorname{Ln} D_{n}^{n+1}\right)(2 M)^{n\left(k_{2}+1\right) /(n+1)}\left(A_{n} c_{n}^{n-1}\right)^{-1} e^{k_{2} l_{2} t}
$$

On the other hand, since $p q \leq\left(k_{1}-\alpha\right)\left(n\left(k_{2}+1\right) /(n+1)-\beta\right)$, there exist two positive constants $l_{1}, l_{2}$ such that

$$
\begin{gathered}
\left(k_{1}-\alpha\right) l_{1} \geq p l_{2}, \quad\left(\frac{n\left(k_{2}+1\right)}{n+1}-\beta\right) l_{2} \geq q l_{1} \\
l_{1} \geq \frac{2 \lambda_{m} R_{1}^{m-k_{1}}}{k_{1}\left(\log R_{2}\right)^{k_{1}} R_{2}^{m}}, \quad l_{2} \geq 2 \bar{\lambda}\left(\lambda_{n}+L n D_{n}^{n+1}\right)(2 M)^{n\left(k_{2}+1\right) /(n+1)}\left(k_{2} A_{n} c_{n}^{n-1}\right)^{-1}
\end{gathered}
$$

By (4.2)-(4.7), it follows that $(\bar{u} ; \bar{v})$ is an upper solution of $(1.1)-(1.3)$. Thus the solutions of (1.1)-(1.3) are global.

Lemma 4.2. Suppose $k_{1}<m, k_{2} \geq n, \alpha \leq k_{1}, \beta \leq n\left(k_{2}+1\right) /(n+1)$ with $p q>\left(k_{1}-\alpha\right)\left(n\left(k_{2}+\right.\right.$ $1) /(n+1)-\beta)$. Then all positive solutions of (1.1)-(1.3) blow up in finite time.

Proof. We first prove that there exist $l_{1} \geq 1, l_{2} \geq 1$ such that

$$
\frac{m k_{1} l_{1}+m}{m-k_{1}} \leq \frac{m \alpha l_{1}+\alpha}{m-k_{1}}+p l_{2}, \quad \frac{n\left(k_{2}+1\right) l_{2}+n}{n+1} \leq \frac{m q l_{1}+q}{m-k_{1}}+\beta l_{2} .
$$


When $\alpha<k_{1}, \beta<n\left(k_{2}+1\right) /(n+1), p q>\left(k_{1}-\alpha\right)\left(n\left(k_{2}+1\right) /(n+1)-\beta\right)$ yields $m\left(k_{1}-\right.$ $\alpha) /\left(\left(m-k_{1}\right) p\right)<m q /\left(\left(m-k_{1}\right)\left(n\left(k_{2}+1\right) /(n+1)-\beta\right)\right)$. Hence there exist $\mu>0$ such that $m\left(k_{1}-\right.$ $\alpha) /\left(\left(m-k_{1}\right) p\right)<\mu<m q /\left(\left(m-k_{1}\right)\left(n\left(k_{2}+1\right) /(n+1)-\beta\right)\right)$. Set $l_{1}=\max \{1,1 / \mu,((m-\alpha) /(m-$ $\left.\left.\left.k_{1}\right)\right) /\left(\mu p-m\left(k_{1}-\alpha\right) /\left(m-k_{1}\right)\right),\left(n /(n+1)-q /\left(m-k_{1}\right)\right) /\left[m q /\left(m-k_{1}\right)-\left(n\left(k_{2}+1\right) /(n+1)-\beta\right) \mu\right]\right\}$, and $l_{2}=\mu l_{1}$.

When $\alpha=k_{1}, \beta \leq n\left(k_{1}+1\right) /(n+1)$, take $l_{2}=\max \left\{1,(m-\alpha) /\left(m-k_{1}\right) p\right\}, l_{1}=$ $\max \left\{1,\left(n /(n+1)-q /\left(m-k_{1}\right)+\left(n\left(k_{2}+1\right) /(n+1)-\beta\right) l_{2}\right)\left(\left(m-k_{1}\right) / m q\right)\right\}$.

When $\alpha \leq k_{1}, \beta=n\left(k_{2}+1\right) /(n+1)$, take $l_{1}=\max \left\{1,\left(n /(n+1)-q /\left(m-k_{1}\right)\right)\left(\left(m-k_{1}\right) / m q\right)\right\}$, $l_{2}=\max \left\{1,\left((m-\alpha) /\left(m-k_{1}\right)+m\left(k_{1}-\alpha\right) l_{1} /\left(m-k_{1}\right)\right)(1 / p)\right\}$.

Let $d_{n}=C_{n}$ if $n<1, d_{n}=D_{n}$ if $n \geq 1$, and $d=\max \{|x| \mid x \in \bar{\Omega}\}, h(x)=\sum_{i=1}^{N} x_{i}+N d+1$, $y=a h^{1+1 / m}(x)+(b-c t)^{-l_{1}}$.

Define $\underline{u}(x, t)=y^{\theta}, \underline{v}=\left(1 /(b-c t)^{l_{2}}\right) \exp \left\{-a \varphi_{n}(x) /(b-c t)^{r}\right\}$, where $\theta=\left(m+1 / l_{1}\right) /(m-$ $\left.k_{1}\right), r=\left(\left(k_{2}-n\right) l_{2}+1\right) /(n+1), b=\max \left\{1,\left((1 / 2) \delta^{1 / \theta}\right)^{-1 / l_{1}}, \delta^{-1 / l_{2}}\right\}$, and

$$
\begin{aligned}
a=\min \left\{b^{-l_{1}}(2 N d+1)^{-(1+1 / m)}, \underline{\Lambda}^{1 / n}\left(B_{n} d_{n}^{n-1}\right)^{-1 / n} b^{-\left(q \theta l_{1}+\beta l_{2}\right) / n},\right. \\
\left.\quad \underline{\Lambda}^{1 / m}\left(\theta^{m}\left(1+\frac{1}{m}\right)^{m} N^{m / 2}(2 N d+1) 2^{m(\theta-1)}\right)^{-1 / m} b^{\left(-\alpha \theta l_{1}+p l_{2}\right) / m}\right\}, \\
c=\min \left\{\frac{n a^{n+1} E_{n}^{n+1}}{k_{2} l_{2}}, \frac{\lambda_{n}\left(k_{2}-n\right) a^{n+1} F_{n}^{n+1}}{k_{2} l_{2}}, \frac{a^{m} \theta^{m-1}(1+1 / m)^{m} N^{(m+1) / 2}}{k_{1} l_{1}}\right\} .
\end{aligned}
$$

By a direct computation, for $(x, t) \in \Omega \times(0, b / c)$, we have

$$
\Delta_{m} \underline{u} \geq\left(a \theta\left(1+\frac{1}{m}\right)\right)^{m} N^{(m+1) / 2} y^{k_{1} \theta-1} y^{m(\theta-1)-k_{1} \theta+1} \geq\left(\underline{u}^{k_{1}}\right)_{t} .
$$

By similar arguments in Lemma 3.2, we have $\left(\underline{v}^{k_{2}}\right)_{t} \leq \Delta_{n} \underline{v}$ for $(x, t) \in \Omega \times(0, b / c)$.

Moreover, for $(x, t) \in \partial \Omega \times(0, b / c)$, we have

$$
\begin{aligned}
\nabla_{m} \underline{u} \cdot \mathcal{v} & \leq\left(a \theta\left(1+\frac{1}{m}\right)\right)^{m} N^{m / 2}(2 N d+1) 2^{m(\theta-1)}(b-c t)^{-m(\theta-1) l_{1}}, \\
\nabla_{n} \underline{v} \cdot \mathcal{v} & \leq \frac{a^{n} B_{n} d_{n}^{n-1}}{(b-c t)^{n\left(l_{2}+r\right)}} ; \\
\underline{u}^{\alpha} \underline{v}^{p} & =\left(a h(x)^{1+1 / m}+(b-c t)^{-l_{1}}\right)^{\theta \alpha}(b-c t)^{-p l_{2}} \geq(b-c t)^{-\left(\alpha \theta l_{1}+p l_{2}\right)}, \\
\underline{u}^{q} \underline{v}^{\beta} & =\left(a h(x)^{1+1 / m}+(b-c t)^{-l_{1}}\right)^{\theta q}(b-c t)^{-\beta l_{2}} \geq(b-c t)^{-\left(q \theta l_{1}+\beta l_{2}\right)} .
\end{aligned}
$$

By (4.8), we have

$$
m(\theta-1) l_{1} \leq \alpha \theta l_{1}+p l_{2}, \quad n\left(l_{2}+r\right) \leq q \theta l_{1}+\beta l_{2} .
$$

By (4.9), (4.12), and (4.13), we have that $(\underline{u}, \underline{v})$ is a lower solution of (1.1)-(1.3), which with the comparison principle implies that the solutions of (1.1)-(1.3) blow up in finite time.

It has been shown from Proposition 2.4 and Lemmas 4.1 and 4.2 that Theorem 1.2 is true. In a similar way to the proof of Theorem 1.2, we have Theorem 1.3. 


\section{Proof of Theorem 1.4}

Lemma 5.1. Suppose $k_{1}<m, k_{2}<n, \alpha \leq k_{1}, \beta \leq k_{2}$ with $p q \leq\left(k_{1}-\alpha\right)\left(k_{2}-\beta\right)$. Then all positive solutions of (1.1)-(1.3) exist globally.

Proof. Take $\bar{u}=a\left(1-\varphi_{m}(x)+e^{l t}\right)^{m /\left(m-k_{1}\right)}, \bar{v}=b\left(1-\varphi_{n}(x)+e^{\theta l t}\right)^{n /\left(n-k_{2}\right)}$, where $\theta=m(n-$ $\left.k_{2}\right)\left(k_{1}-\alpha\right) / n\left(m-k_{1}\right) p$ and $a, b, l$ are the undetermined positive constants.

Calculating directly for $(x, t) \in \Omega \times \mathbb{R}^{+}$, we have by Lemma 2.8 that

$$
\begin{aligned}
\left(\bar{u}^{k_{1}}\right)_{t} \geq & \frac{a^{k_{1}} m k_{1} l}{2\left(m-k_{1}\right)}\left(1-\varphi_{m}(x)+e^{l t}\right)^{m k_{1} /\left(m-k_{1}\right)} \\
\geq & \frac{a^{k_{1}} m k_{1} l}{2\left(m-k_{1}\right)} f_{1}\left(\frac{m k_{1}}{m-k_{1}}\right)\left(1-\varphi_{m}(x)+e^{m k_{1} l t /\left(m-k_{1}\right)}\right) \\
\Delta_{m} \bar{u} \leq & \lambda_{m} \varphi_{m}^{m}\left(\frac{a m}{m-k_{1}}\right)^{m} f_{2}\left(\frac{m k_{1}}{m-k_{1}}\right)\left(1-\varphi_{m}(x)+e^{m k_{1} l t /\left(m-k_{1}\right)}\right) \\
& +\left(\frac{a m}{m-k_{1}}\right)^{m} \frac{m k_{1}}{m-k_{1}} D_{m}^{m+1} f_{2}\left(\frac{m k_{1}}{m-k_{1}}\right)\left(1-\varphi_{m}(x)+e^{m k_{1} l t /\left(m-k_{1}\right)}\right) \\
\leq & \left(\lambda_{m}+\frac{m k_{1}}{m-k_{1}} D_{m}^{m+1}\right)\left(\frac{a m}{m-k_{1}}\right)^{m} f_{2}\left(\frac{m k_{1}}{m-k_{1}}\right)\left(1-\varphi_{m}(x)+e^{m k_{1} l t /\left(m-k_{1}\right)}\right) \\
\left(\bar{v}^{k_{2}}\right)_{t} \geq & \frac{b^{k_{2}} n k_{2} \theta l}{2\left(n-k_{2}\right)}\left(1-\varphi_{n}(x)+e^{\theta l t}\right)^{n k_{2} /\left(n-k_{2}\right)} \\
\geq & \frac{b^{k_{2}} n k_{2} \theta l}{2\left(n-k_{2}\right)} f_{1}\left(\frac{n k_{2}}{n-k_{2}}\right)\left(1-\varphi_{n}(x)+e^{n k_{2} \theta l t /\left(n-k_{2}\right)}\right) \\
\Delta_{n} \bar{v} \leq & \left(\lambda_{n}+\frac{n k_{2}}{n-k_{2}} D_{n}^{n+1}\right)\left(\frac{b n}{n-k_{2}}\right)^{n} f_{2}\left(\frac{n k_{2}}{n-k_{2}}\right)\left(1-\varphi_{n}(x)+e^{n k_{2} \theta l t /\left(n-k_{2}\right)}\right) .
\end{aligned}
$$

Let $c_{m}=C_{m}$ if $m \geq 1, c_{m}=D_{m}$ if $m<1$, and $c_{n}=C_{n}$ if $n \geq 1, c_{n}=D_{n}$ if $n<1$. We have on the boundary that

$$
\begin{aligned}
\nabla_{m} \bar{u} \cdot v & \geq\left(\frac{a m}{m-k_{1}}\right)^{m} c_{m}^{m-1} A_{m}\left(1-\varphi_{m}(x)+e^{l t}\right)^{m k_{1} /\left(m-k_{1}\right)} \\
& \geq a^{m-\alpha}\left(\frac{m}{m-k_{1}}\right)^{m} c_{m}^{m-1} A_{m} f_{1}\left(\frac{m\left(k_{1}-\alpha\right)}{m-k_{1}}\right)\left(1+e^{m\left(k_{1}-\alpha\right) l t /\left(m-k_{1}\right)}\right) \bar{u}^{\alpha}, \\
\nabla_{n} \bar{v} \cdot v & \geq b^{n-\beta}\left(\frac{n}{n-k_{2}}\right)^{n} c_{n}^{n-1} A_{n} f_{1}\left(\frac{n\left(k_{2}-\beta\right)}{n-k_{2}}\right)\left(1+e^{n\left(k_{2}-\beta\right) \theta l t /\left(n-k_{2}\right)}\right)^{\beta} \\
& \geq b^{n-\beta}\left(\frac{n}{n-k_{2}}\right)^{n} c_{n}^{n-1} A_{n} f_{1}\left(\frac{n\left(k_{2}-\beta\right)}{n-k_{2}}\right)\left(1+e^{m q l t /\left(m-k_{1}\right)}\right) \bar{v}^{\beta} ; \\
\bar{u}^{\alpha} \bar{v}^{p} & =b^{p}\left(1+e^{\theta l t}\right)^{n p /\left(n-k_{2}\right)} \bar{u}^{\alpha} \leq b^{p} f_{2}\left(\frac{n p}{n-k_{2}}\right)\left(1+e^{m\left(k_{1}-\alpha\right) l t /\left(m-k_{1}\right)}\right) \bar{u}^{\alpha}, \\
\bar{u}^{q} \bar{v}^{\beta} & =a^{q}\left(1+e^{l t}\right)^{m q /\left(m-k_{1}\right)} \bar{v}^{\beta} \leq a^{q} f_{2}\left(\frac{m q}{m-k_{1}}\right)\left(1+e^{m q l t /\left(m-k_{1}\right)}\right) \bar{v}^{\beta} .
\end{aligned}
$$


Since $p q \leq\left(k_{1}-\alpha\right)\left(k_{2}-\beta\right)<(m-\alpha)(n-\beta)$, we know by Lemma 2.8 that there exist constants $a \geq\left\|u_{0}(x)\right\|_{\infty}, b \geq\left\|v_{0}(x)\right\|_{\infty}$ such that

$$
\begin{aligned}
a^{m-\alpha}\left(\frac{m}{m-k_{1}}\right)^{m} c_{m}^{m-1} A_{m} f_{1}\left(\frac{m\left(k_{1}-\alpha\right)}{m-k_{1}}\right) & \geq \bar{\lambda} b^{p} f_{2}\left(\frac{n p}{n-k_{2}}\right), \\
b^{n-\beta}\left(\frac{n}{n-k_{2}}\right)^{n} c_{n}^{n-1} A_{n} f_{1}\left(\frac{n\left(k_{2}-\beta\right)}{n-k_{2}}\right) & \geq \bar{\lambda} a^{q} f_{2}\left(\frac{m q}{m-k_{1}}\right) .
\end{aligned}
$$

For the above constants $a, b$, we choose a constant $l$ so large that

$$
\begin{gathered}
\left(\lambda_{m}+\frac{m k_{1}}{m-k_{1}} D_{m}^{m+1}\right)\left(\frac{a m}{m-k_{1}}\right)^{m} f_{2}\left(\frac{m k_{1}}{m-k_{1}}\right) \leq \frac{a^{k_{1}} m k_{1} l}{2\left(m-k_{1}\right)} f_{1}\left(\frac{m k_{1}}{m-k_{1}}\right), \\
\left(\lambda_{n}+\frac{n k_{2}}{n-k_{2}} D_{n}^{n+1}\right)\left(\frac{b n}{n-k_{2}}\right)^{n} f_{2}\left(\frac{n k_{2}}{n-k_{2}}\right) \leq \frac{b^{k_{2}} n k_{2} \theta l}{2\left(n-k_{2}\right)} f_{1}\left(\frac{n k_{2}}{n-k_{2}}\right) .
\end{gathered}
$$

By (5.1)-(5.4), we know that $(\bar{u}, \bar{v})$ is an upper solution of (1.1)-(1.3), Thus the solutions of (1.1)-(1.3) are global.

Lemma 5.2. Suppose $k_{1}<m, k_{2}<n, \alpha \leq k_{1}, \beta \leq k_{2}$ with $p q>\left(k_{1}-\alpha\right)\left(k_{2}-\beta\right)$. Then all positive solutions of (1.1)-(1.3) blow up in finite time.

Proof. We first prove that there exist $l_{1} \geq 1, l_{2} \geq 1$ such that

$$
\frac{m k_{1} l_{1}+m}{m-k_{1}} \leq \frac{m \alpha l_{1}+\alpha}{m-k_{1}}+\frac{n p l_{2}+p}{n-k_{2}}, \quad \frac{n k_{2} l_{2}+n}{n-k_{2}} \leq \frac{m q l_{1}+q}{m-k_{1}}+\frac{n \beta l_{2}+\beta}{n-k_{2}} .
$$

In fact, when $\alpha<k_{1}, \beta<k_{2}, p q>\left(k_{1}-\alpha\right)\left(k_{2}-\beta\right)$ yields $\left(m\left(k_{1}-\alpha\right) /\left(m-k_{1}\right)\right)\left(\left(n-k_{2}\right) / n p\right)<$ $\left(m q /\left(m-k_{1}\right)\right)\left(\left(n-k_{2}\right) / n\left(k_{2}-\beta\right)\right)$. Hence there exists $\mu>0$ such that $\left(m\left(k_{1}-\alpha\right) /\left(m-k_{1}\right)\right)((n-$ $\left.\left.k_{2}\right) / n p\right)<\mu<\left(m q /\left(m-k_{1}\right)\right)\left(\left(n-k_{2}\right) / n\left(k_{2}-\beta\right)\right)$. Set $l_{1}=\max \left\{1,1 / \mu,\left((m-\alpha) /\left(m-k_{1}\right)-\right.\right.$ $\left.p /\left(n-k_{2}\right)\right) /\left(\left(n p /\left(n-k_{2}\right)\right) \mu-m\left(k_{1}-\alpha\right) /\left(m-k_{1}\right)\right),\left((n-\beta) /\left(n-k_{2}\right)-q /\left(m-k_{1}\right)\right) /(m q /(m-$ $\left.\left.\left.k_{1}\right)-\left(n\left(k_{2}-\beta\right) /\left(n-k_{2}\right)\right) \mu\right)\right\}$, and $l_{2}=\mu l_{1}$.

When $k_{1} \leq \alpha$ and $\beta=k_{2}$, take $l_{1}=\max \left\{1,\left((n-\beta) /\left(n-k_{2}\right)-q /\left(m-k_{1}\right)\right)\left(\left(m-k_{1}\right) / m q\right)\right\}, l_{2}=$ $\max \left\{1,\left((m-\alpha) /\left(m-k_{1}\right)-p /\left(n-k_{2}\right)+m\left(k_{1}-\alpha\right) l_{1} /\left(m-k_{1}\right)\right)\left(\left(n-k_{2}\right) / n p\right)\right\}$.

When $k_{1}=\alpha$ and $\beta \leq k_{2}$, let $l_{2}=\max \left\{1,\left((m-\alpha) /\left(m-k_{1}\right)-p /\left(n-k_{2}\right)\right)\left(\left(n-k_{2}\right) / n p\right)\right\}, l_{1}=$ $\max \left\{1,\left((n-\beta) /\left(n-k_{2}\right)-q /\left(m-k_{1}\right)+n\left(k_{2}-\beta\right) l_{2} /\left(n-k_{2}\right)\right)\left(\left(m-k_{1}\right) / m q\right)\right\}$.

Take $y=a h^{1+1 / m}(x)+(b-c t)^{-l_{1}}, z=a h^{1+1 / n}(x)+(b-c t)^{-l_{2}}$, and $\underline{u}=y^{\theta}, \underline{v}=z^{\sigma}$, where $\theta=\left(m+1 / l_{1}\right) /\left(m-k_{1}\right), \sigma=\left(n+1 / l_{2}\right) /\left(n-k_{2}\right), b=\max \left\{1,\left((1 / 2) \delta^{1 / \theta}\right)^{-1 / l_{1}},\left((1 / 2) \delta^{1 / \sigma}\right)^{-1 / l_{2}}\right\}$, and

$$
\begin{aligned}
& a=\min \left\{b^{-l_{1}}(2 N d+1)^{-(1+m) / m},\left(\underline{\lambda}^{-1}\left[\frac{(1+m) \theta N^{1 / 2} 2^{\theta-1}}{m}\right]^{m}(2 N d+1)\right)^{-1 / m} b^{-\left(\alpha \theta l_{1}+p \sigma l_{2}\right) / m}\right. \\
&\left.b^{-l_{2}}(2 N d+1)^{-(1+n) / n},\left(\underline{\lambda}^{-1}\left[\frac{(1+n) \sigma N^{1 / 2} 2^{\sigma-1}}{n}\right]^{n}(2 N d+1)\right)^{-1 / n} b^{-\left(q \theta l_{1}+\beta \sigma l_{2}\right) / n}\right\} \\
& c=\left.\min \left\{\frac{a^{m} \theta^{m-1}(1+1 / m)^{m} N^{(m+1) / 2}}{k_{1} l_{1}}, \frac{a^{n} \sigma^{n-1}(1+1 / n)^{n} N^{(n+1) / 2}}{k_{2} l_{2}}\right\} .6\right)
\end{aligned}
$$


By a direct computation for $(x, t) \in \Omega \times(0, b / c)$, we have

$$
\begin{aligned}
& \Delta_{m} \underline{u} \geq\left(a \theta\left(1+\frac{1}{m}\right)\right)^{m} N^{(m+1) / 2} y^{k_{1} \theta-1} y^{m(\theta-1)-k_{1} \theta+1} \geq\left(\underline{u}^{k_{1}}\right)_{t^{\prime}} \\
& \Delta_{n} \underline{\boldsymbol{v}} \geq\left(a \sigma\left(1+\frac{1}{n}\right)\right)^{n} N^{(n+1) / 2} y^{k_{2} \sigma-1} y^{n(\sigma-1)-k_{2} \sigma+1} \geq\left(\underline{\boldsymbol{v}}^{k_{2}}\right)_{t} .
\end{aligned}
$$

For $(x, t) \in \partial \Omega \times(0, b / c)$, we have

$$
\begin{aligned}
\nabla_{m} \underline{u} \cdot \mathcal{v} & \leq\left(a \theta\left(1+\frac{1}{m}\right)\right)^{m} N^{m / 2}(2 N d+1) 2^{m(\theta-1)}(b-c t)^{-m(\theta-1) l_{1}}, \\
\nabla_{n} \underline{v} \cdot \mathcal{v} & \leq\left(a \sigma\left(1+\frac{1}{n}\right)\right)^{n} N^{n / 2}(2 N d+1) 2^{n(\sigma-1)}(b-c t)^{-n(\sigma-1) l_{2}} \\
\underline{u}^{\alpha} \underline{v}^{p} & =y^{\alpha \theta} z^{p \sigma} \geq(b-c t)^{-\left(\alpha \theta l_{1}+p \sigma l_{2}\right)}, \\
\underline{u}^{q} \underline{v}^{\beta} & =y^{q \theta} z^{\beta \sigma} \geq(b-c t)^{-\left(q \theta l_{1}+\beta \sigma l_{2}\right)} .
\end{aligned}
$$

Moreover, (5.5) implies

$$
m(\theta-1) l_{1} \leq \alpha \theta l_{1}+p \sigma l_{2}, \quad n(\sigma-1) l_{2} \leq q \theta l_{1}+\beta \sigma l_{2} .
$$

It follows from $(5.6),(5.8)-(5.11)$ that $(\underline{u}, \underline{v})$ is a lower solution of $(1.1)-(1.3)$. Because $(\underline{u}, \underline{v})$ blows up in finite time, and so does $(u, v)$.

By Proposition 2.6 and Lemmas 5.1 and 5.2, we see that Theorem 1.4 holds.

\section{Acknowledgments}

This work was partially supported by NNSF of China (10771226), was partially supported by Natural Science Foundation Project of CQ CSTC (2007BB0124), and was partially supported by Natural Science Foundation Project of China West Normal University (07B047).

\section{References}

[1] Z. Tan and X. G. Liu, "Non-Newton filtration equation with nonconstant medium void and critical Sobolev exponent," Acta Mathematica Sinica, vol. 20, no. 2, pp. 367-378, 2004.

[2] S. Wang, "Doubly nonlinear degenerate parabolic systems with coupled nonlinear boundary conditions," Journal of Differential Equations, vol. 182, no. 2, pp. 431-469, 2002.

[3] Z. Wang, J. Yin, and C. Wang, "Critical exponents of the non-Newtonian polytropic filtration equation with nonlinear boundary condition," Applied Mathematics Letters, vol. 20, no. 2, pp. 142-147, 2007.

[4] Z. Wu, J. Zhao, J. Yin, and H. Li, Nonlinear Diffusion Equations, World Scientific, River Edge, NJ, USA, 2001.

[5] Z. Yang and Q. Lu, "Nonexistence of positive solutions to a quasilinear elliptic system and blow-up estimates for a non-Newtonian filtration system," Applied Mathematics Letters, vol. 16, no. 4, pp. 581587, 2003.

[6] Z. Yang and Q. Lu, "Blow-up estimates for a quasi-linear reaction-diffusion system," Mathematical Methods in the Applied Sciences, vol. 26, no. 12, pp. 1005-1023, 2003.

[7] X. Song and S. Zheng, "Blow-up analysis for a quasilinear parabolic system with multi-coupled nonlinearities," Journal of Mathematical Analysis and Applications, vol. 281, no. 2, pp. 739-756, 2003. 
[8] M. Wang and $\mathrm{Y}$. Wu, "Global existence and blow-up problems for quasilinear parabolic equations with nonlinear boundary conditions," SIAM Journal on Mathematical Analysis, vol. 24, no. 6, pp. 1515-1521, 1993.

[9] M. Wang, "The long-time behavior of solutions to a class of quasilinear parabolic equations with nonlinear boundary conditions," Acta Mathematica Sinica, vol. 39, no. 1, pp. 118-124, 1996.

[10] N. Wolanski, "Global behavior of positive solutions to nonlinear diffusion problems with nonlinear absorption through the boundary," SIAM Journal on Mathematical Analysis, vol. 24, no. 2, pp. 317-326, 1993.

[11] M. Wang and S. Wang, "Quasilinear reaction-diffusion systems with nonlinear boundary conditions," Journal of Mathematical Analysis and Applications, vol. 231, no. 1, pp. 21-33, 1999.

[12] M. Wang, "Fast-slow diffusion systems with nonlinear boundary conditions," Nonlinear Analysis: Theory, Methods E Applications, vol. 46, no. 6, pp. 893-908, 2001.

[13] W. J. Sun and S. Wang, "Nonlinear degenerate parabolic equation with nonlinear boundary condition," Acta Mathematica Sinica, vol. 21, no. 4, pp. 847-854, 2005.

[14] H. W. Alt and S. Luckhaus, "Quasilinear elliptic-parabolic differential equations," Mathematische Zeitschrift, vol. 183, no. 3, pp. 311-341, 1983.

[15] P. Lindqvist, "On the equation $\operatorname{div}\left(|\nabla u|^{p-2} \nabla u\right)+\lambda|u|^{p-2} u=0$," Proceedings of the American Mathematical Society, vol. 109, no. 1, pp. 157-164, 1990.

[16] P. Lindqvist, "Addendum to on the equation $\operatorname{div}\left(|\nabla u|^{p-2} \nabla u\right)+\lambda|u|^{p-2} u=0$," Proceedings of the American Mathematical Society, vol. 116, no. 2, pp. 583-584, 1992.

[17] Y. Li and C. Xie, "Blow-up for $p$-Laplacian parabolic equations," Electronic Journal of Differential Equations, vol. 2003, no. 20, pp. 1-12, 2003. 